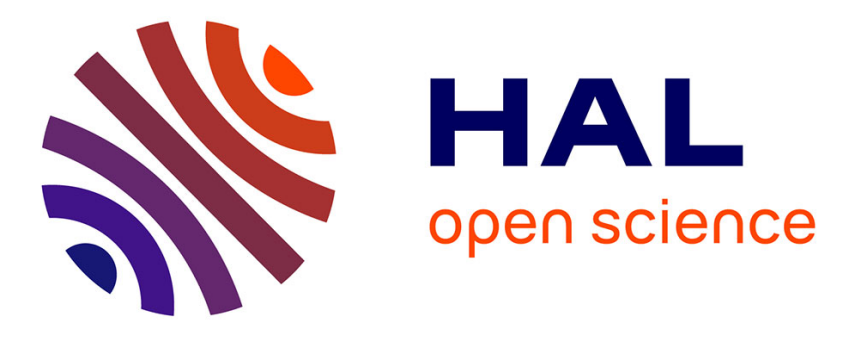

\title{
The Joule heating problem in silver nanowire transparent electrodes
}

H. H. Khaligh, L. Xu, Alireza Khosropour, Alexandra Madeira, M. Romano, Christophe Pradére, Mona Tréguer-Delapierre, Laurent Servant, Michael A. Pope, Irene A. Goldthorpe

\section{To cite this version:}

H. H. Khaligh, L. Xu, Alireza Khosropour, Alexandra Madeira, M. Romano, et al.. The Joule heating problem in silver nanowire transparent electrodes. Nanotechnology, 2017, 28 (42), pp.425703. 10.1088/1361-6528/aa7f34. hal-01630585

\section{HAL Id: hal-01630585 https://hal.science/hal-01630585}

Submitted on 5 May 2021

HAL is a multi-disciplinary open access archive for the deposit and dissemination of scientific research documents, whether they are published or not. The documents may come from teaching and research institutions in France or abroad, or from public or private research centers.
L'archive ouverte pluridisciplinaire HAL, est destinée au dépôt et à la diffusion de documents scientifiques de niveau recherche, publiés ou non, émanant des établissements d'enseignement et de recherche français ou étrangers, des laboratoires publics ou privés. 


\title{
The Joule heating problem in silver nanowire transparent electrodes
}

\author{
H H Khaligh ${ }^{1,2}$, L X $\mathbf{u}^{2,3}$, A Khosropour ${ }^{1,2}$, A Madeira ${ }^{1,2,4}$, M Romano $^{5}$, \\ C Pradére ${ }^{5}$, M Tréguer-Delapierre ${ }^{4}$, L Servant ${ }^{6}$, M A Pope $^{2,3}$ and \\ I A Goldthorpe ${ }^{1,2}$ \\ ${ }^{1}$ Department of Electrical \& Computer Engineering, University of Waterloo, Waterloo, ON N2L 3G1, \\ Canada \\ ${ }^{2}$ Waterloo Institute for Nanotechnology, University of Waterloo, Waterloo, ON N2L 3G1, Canada \\ ${ }^{3}$ Department of Chemical Engineering, University of Waterloo, Waterloo, ON N2L 3G1, Canada \\ ${ }^{4}$ ICMCB CNRS, Université de Bordeaux, F 33608 Pessac Cedex, France \\ ${ }^{5}$ I2M, Département TREFLE, UMR CNRS 5295 site ENSAM, F 33405 Talence Cedex, France \\ ${ }^{6}$ Institut des Sciences Moléculaires, Université de Bordeaux, F 33405 Talence Cedex, France \\ E mail: igoldthorpe@uwaterloo.ca
}

\begin{abstract}
Silver nanowire transparent electrodes have shown considerable potential to replace conventional transparent conductive materials. However, in this report we show that Joule heating is a unique and serious problem with these electrodes. When conducting current densities encountered in organic solar cells, the average surface temperature of indium tin oxide (ITO) and silver nanowire electrodes, both with sheet resistances of $60 \mathrm{ohms} /$ square, remains below $35^{\circ} \mathrm{C}$. However, in contrast to ITO, the temperature in the nanowire electrode is very non-uniform, with some localized points reaching temperatures above $250{ }^{\circ} \mathrm{C}$. These hotspots accelerate nanowire degradation, leading to electrode failure after 5 days of continuous current flow. We show that graphene, a commonly used passivation layer for these electrodes, slows nanowire degradation and creates a more uniform surface temperature under current flow. However, the graphene does not prevent Joule heating in the nanowires and local points of high temperature ultimately shift the failure mechanism from nanowire degradation to melting of the underlying plastic substrate. In this paper, surface temperature mapping, lifetime testing under current flow, post-mortem analysis, and modelling illuminate the behaviour and failure mechanisms of nanowires under extended current flow and provide guidelines for managing Joule heating.
\end{abstract}

Supplementary material for this article is available online

Keywords: transparent electrode, silver nanowire, Joule heating, reduced graphene oxide

(Some figures may appear in colour only in the online journal)

\section{Introduction}

Silver nanowire (AgNW) transparent electrodes have emerged as an alternative material to conventional transparent conductive oxides. They can exhibit similar conductivity and transparency values as indium tin oxide (ITO) films [1, 2], the most common transparent conductive material, while being lower in cost. Furthermore, nanowire electrodes have the advantages of being deposited on substrates using low temperature solution deposition methods and are able to maintain their conductivity after repeated bending. These aspects make them particularly desirable for use on plastic substrates for flexible electronics, for which ITO is inappropriate [3].

AgNW transparent electrodes are already being used in commercial touchscreen applications. These touchscreens work by creating and changing an electric field between the nanowire electrode and a parallel electrode, with an active 
dielectric material in between that responds to the field. A current flows across the nanowire electrode only for a short time to change its potential during switching, with minimal current flowing otherwise. This is also true for other capacitive-type devices such as smart windows [4]. However, for devices such as solar cells, organic light emitting diodes (OLEDs), and transparent heaters, there are long periods of time when current continuously flows across the transparent electrode during device operation, resulting in Joule heating. Although not typically problematic for ITO electrodes, we have previously shown that the Joule heating created at the current levels typically encountered in organic solar cells causes nanowire electrodes to fail in a matter of days. The nanowires breakup and electrical discontinuities appear in the electrode [5].

Multiple mechanisms can lead to silver nanowire degradation. Silver nanowires corrode in air, specifically due to the presence of trace amounts of sulfur [6], and this corrosion phenomenon is accelerated at elevated temperatures [7]. Additionally, silver nanowires are not morphologically stable owing, in part, to their high surface-area volume ratio. They tend to break into small segments, a behaviour usually attributed to the Rayleigh instability [8 10$]$. Like corrosion, this instability of metal nanowires is also worsened at higher temperatures [10]. In order to slow nanowire degradation and thus increase the lifetime of $\mathrm{AgNW}$ electrodes, various passivation strategies have been developed that use an outer layer to protect the silver nanowires from corrosion and other side reactions with the environment. The passivation layer must be optically transparent, and electrically conductive as well if it stands, for example, between the nanowire network and the active part of a solar cell. Passivation materials such as zinc oxide $(\mathrm{ZnO})$, titanium oxide $\left(\mathrm{TiO}_{2}\right)$, and graphene have been used [11 14]. Reduced graphene oxide (RGO), in particular, is attractive since it can act as a barrier for both moisture and gases in air [15]. It can be deposited as films thinner than $\mathrm{ZnO}$ and $\mathrm{TiO}_{2}$ ones, optimizing optical transparency. Furthermore, like AgNW films, RGO is mechanically flexible and can be deposited from solution. Several studies have demonstrated the utility of RGO as a passivation layer for AgNW electrodes $[13,1619]$. For instance, whereas the sheet resistance of an unpassivated $\mathrm{AgNW}$ electrode more than tripled in less than a week while annealed at $70{ }^{\circ} \mathrm{C}$ in air, the resistance of the same nanowire electrode passivated with a uniformly dip-coated RGO layer increased by less than $50 \%$ over the same time [20]. If a layer of graphene is instead deposited on a AgNW electrode using chemical vapour deposition (CVD), it can stabilize the silver nanowires more effectively. A recent experiment revealed only a $10 \%$ increase of sheet resistance after one month while annealed at $70{ }^{\circ} \mathrm{C}$ [21]. However, this latter method is presently not economical for commercial manufacturing, especially since the graphene layer cannot be directly synthesized on the nanowires but rather needs to be first deposited on a substrate and then transferred onto the nanowire film.

In the above studies, degradation of passivated and unpassivated silver nanowire electrodes were evaluated either at room temperature or when the electrode was annealed in an oven (or on a hotplate) to achieve an elevated temperature. In all cases, the temperature was the same at all points on the electrode. However, the surface temperature profile across a nanowire electrode is not uniform when it is used in a solar cell, OLED, or tranparent heater. Unlike in a continuous conducting film (e.g. ITO) where current flows throughout the entire area of the film, in the case of nanowire electrodes current only flows through the thin metal pathways. Furthermore, there are variations in the nanowire density across the film and thus the current density flowing in some nanowire pathways is higher than in others. These factors combined result in a non-uniform temperature distribution in the electode where the temperatures of some individual nanowires are significantly higher than the average surface temperature of the electrode [2]. In this work we investigate AgNW electrodes and RGO-passivated AgNW electrodes under current flow. Compared to annealing, this is a more realistic situation for studying the performance and lifetime of the electrodes when they are operated in a solar cell or OLED. As other reports have shown, we confirm that a RGO passivation layer does increase lifetimes by slowing nanowire degradation during an anneal. However, when the nanowire electrode is instead under current flow, RGO is not nearly as effective as it does not prevent Joule heating. It is shown that the Joule heating in RGO-passivated electrodes results in local melting and deformation of the plastic substrate leading ultimately to electrode failure. These results indicate that Joule heating is an issue that cannot be overlooked and needs to be effectively addressed and managed if nanowire electrodes are to be used in devices such as solar cells, OLEDs, and transparent heaters.

\section{Experimental section}

\subsection{Nanowire electrode fabrication}

Silver nanowires dispersed in ethanol were purchased from Blue Nano Inc. (Charlotte, NC) with an average diameter and length of $90 \mathrm{~nm}$ and $25 \mu \mathrm{m}$, respectively. Polyethylene terephthalate (PET) films (127 $\mu \mathrm{m}$ thick from Dupont Tianjin Inc.) were used as substrates for the electrodes (PET was used rather than glass since a distinct advantage of AgNW electrodes is their suitability for plastic substrates). The PET films were cleaned sequentially in a sonication bath of acetone, isopropanol alcohol, and distilled water each for $1 \mathrm{~min}$, then dried with nitrogen gas. The nanowires were deposited on the PET films using the Mayer rod coating technique to obtain a random network of silver nanowires [22]. The active area of the deposited AgNW film was $2 \mathrm{~cm} \times 2 \mathrm{~cm}$ with two strips of $0.5 \mathrm{~cm}$ wide copper tape installed at the two ends of the electrodes $(2 \mathrm{~cm}$ apart $)$ as current collectors. The transparency and conductivity of the silver nanowire electrodes can be controlled by changing the concentration of the nanowires in ethanol and the number of coating layers. The dependency of the transmitance and conductivity has been reported elsewhere [23]. The deposited films were dried in air for $5 \mathrm{~min}$ and mechanically pressed by rollers at room temperature to reduce the nanowire junction resistances [24]. 
ITO on PET with a sheet resistance of $60 \mathrm{ohms} / \mathrm{sq}$ was purchased from Sigma Aldrich. Although lower sheet resistances are desired for solar cell applications, ITO on PET has higher resistance than on glass due to the need for lower deposition temperatures. To be able to compare with ITO, the AgNW electrodes were also made with a sheet resistance of $60 \pm 10 \% \mathrm{ohms} / \mathrm{sq}$. AgNW electrodes with sheet resistances of $20 \mathrm{ohms} / \mathrm{sq}$ were prepared as well to see the effect of Joule heating in lower resistance networks.

\subsection{Determining current value}

The current density assumed in this study was $20 \mathrm{~mA} \mathrm{~cm}^{2}$, which is the typical operating current of the best performing organic solar cells in the literature $[25,26]$. Current densities in OLEDs can also reach values as high as $20 \mathrm{~mA} \mathrm{~cm}^{2}$ [27]. The maximum current is usually incurred near the current collector bar in these devices. In common designs of organic solar cell modules the width of the active layer is around $1 \mathrm{~cm}$, and the current flowing through the transparent electrode is collected at one end only (see figure S1, available online at stacks.iop.org/ NANO/28/425703/mmedia) [28 30]. Therefore, with a current density of $20 \mathrm{~mA} \mathrm{~cm}{ }^{2}$, the current at the current collector bar will reach $20 \mathrm{~mA}$ per centimetre length $\left(20 \mathrm{~mA} \mathrm{~cm}^{1}\right)$ of current collector. As this is the typical maximum current incurred in the transparent electrode, this is the value we used in the experiments.

\subsection{Graphene oxide (GO) monolayer fabrication}

GO was first prepared using the Tour method [31]. A continuous, densely tiled, monolayer of the GO was then formed on the surface of water using a modified Langmuir Blodgett (LB) trough technique [32]. Instead of using mechanical barriers to compress the film, as in the typical LB process, the spreading force of the solvent is used to compress the films against the edge of the trough. Thus, in principle, this approach has the potential to be adapted to a roll-to-roll manufacturing by withdrawing a substrate during material deposition. Such a system will be reported elsewhere. The asprepared AgNW-coated PET samples were placed at the bottom of the trough prior to GO deposition, and the floating monolayer GO film was lowered onto the substrates by slowly draining the water. In addition to coating PET samples, mica and highly oriented pyrolytic graphite (HOPG) substrates were coated at the same time to verify film morphology and coverage using atomic force microscopy (AFM) and scanning electron microscopy (SEM). The GO was chemically reduced using $\mathrm{NaBH}_{4}$. Further details of the GO synthesis, monolayer formation, and chemical reduction can be found in the supplementary information.

\subsection{Characterization}

Contact mode AFM was carried out on GO films deposited on atomically flat muscovite mica (SPI) substrates using NPSTT10 tips (Bruker) and a Nanoscope MultiMode AFM (Veeco). AFM images were analysed using Gwyddion software to extract height profiles between the atomically smooth substrate and the GO sheets, and a custom MATLAB code was used to estimate sheet thicknesses from the steps in the height profiles.

The sheet resistance of the electrodes with and without the RGO layer was measured by either a multimeter or a 4-point probe measurement system. UV vis photospectroscopy with an integrating sphere was used to measure the transparency of the electrodes. SEM images were taken before and after failure of the electrodes.

A DC power supply was connected to the current collectors to apply a constant current of $20 \mathrm{~mA} \mathrm{~cm}^{1}$ across the electrodes. A multimeter and a flat leaf-style thermocouple were used to continuously monitor the voltage across the electrode and its average surface temperature, respectively, during extended time experiments and PC link software was used to record the data (a schematic of the experimental setup is shown in figure S2). In a separate measurement, an infrared (IR) camera was used to map the spatial temperature distribution over the electrode area while the electrode was conducting $20 \mathrm{~mA} \mathrm{~cm}^{1}$ of current. The IR camera (FLIR SC7000) had an indium antimony detector composed of a focal plane array featuring 81920 pixels (matrix $320 \times 256$ ), and a simultaneous measurement at all 81920 spots could be performed using the snapshot mode. The camera was equipped with an IR lens with a focal length of $25 \mathrm{~mm}$. The resulting spatial resolution per pixel is around $250 \mu \mathrm{m} \times 250 \mu \mathrm{m}$. A previously reported calibration method $[32,33]$ was used to retrieve the absolute temperature of the samples (semi-transparent samples) from the raw intensity data provided by the IR camera. More details about the calibration can be found in the supplementary information.

\subsection{Modelling}

Random networks of silver nanowires were modelled in MATLAB. The nanowires were modelled as cylinders having the same average diameter and length as the nanowires used in our experiment $(90 \mathrm{~nm}$ and $25 \mu \mathrm{m}$, respectively). The resistivity of the nanowires considered in the model was set to $27 \mathrm{n} \Omega \mathrm{m}$, which is the average resistivity reported for $90 \mathrm{~nm}$ diameter silver nanowires [34 36]. The nanowires were randomly distributed over a $300 \mu \mathrm{m} \times 300 \mu \mathrm{m}$ area (figure 5(a)) and treated as pure resistors. The density of nanowires was chosen to generate nanowire networks with sheet resistances of $60 \pm 15 \% \mathrm{ohms} / \mathrm{sq}$. The overall circuit was analysed using HSPICE. An HSPICE netlist was constructed for this random nanowire network in order to calculate the sheet resistance of the modelled network and extract the electrical current in individual nanowires.

The electric current values retrieved from HSPICE simulations were subsequently used to model Joule heating in individual nanowires using the Joule heating module of COMSOL Multiphysics software. The temperature profile of nanowires under the range of current densities were simulated for wires lying on a PET substrate with and without a RGO passivation layer. The graphene sheets are much larger than the diameter of the nanowires and cover their surface and the surrounding substrate conformably, as shown by others [19]. 


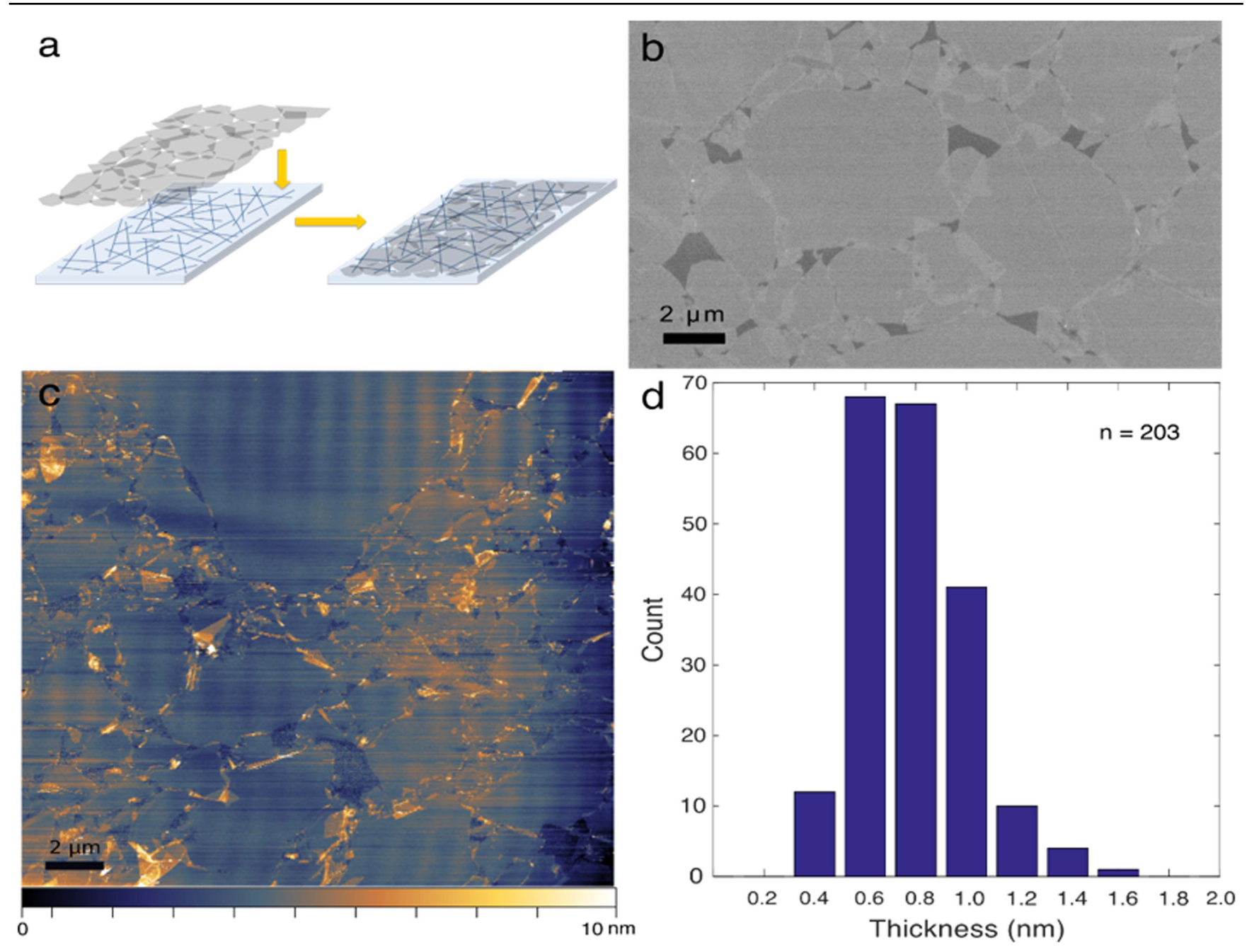

Figure 1. Reduced graphene oxide blocking layer. (a) Schematic illustrating graphene oxide monolayer deposition on a silver nanowire/PET film. (b) SEM image showing contrast between the graphene oxide film (light) and some pinholes exposing the HOPG substrate (dark). (c) Contact mode AFM image of the graphene oxide film on mica. (d) Histogram of graphene oxide sheet thickness estimated from AFM imaging.

In the COMSOL model the conformal contact was simplified to square shape. This shape has three points of contact with the nanowire with small gaps between the nanowire and the RGO layer which is similar to the real structure. The area of the substrate was selected according to the experimental nanowire density by dividing the area of the substrate by the number of nanowires. Heat convection was considered at the top and bottom of the PET sample and all surfaces of the nanowires and the RGO layer as the boundary condition. More details about modelling can be found in the supplementary information.

\section{Results and discussion}

\subsection{Characterization of electrodes}

Our first goal was to create a transparent barrier film of RGO to protect the $\mathrm{Ag}$ nanowires as illustrated schematically in figure 1. The morphology and coverage of the RGO film, deposited by a modified LB technique, are shown in the SEM and AFM images in figures 1(b) and (c), respectively. The contrast in the SEM image (figure 1(b)) shows the RGO film (bright) and the underlying HOPG substrate (dark) and indicates that the film is composed of densely tiled and partially overlapping (even brighter contrast regions) sheets. The film coverage, estimated by pixel counting, is over $95 \%$. The AFM images of films deposited on mica (figure 1(c)) show the same coverage. A histogram generated by analysing AFM thickness profiles of over 200 sheets is shown in figure 1(d) and indicates that the tiled sheets have a narrow distribution of thicknesses centred around $0.7 \mathrm{~nm}$. This indicates that the film is composed almost entirely of monolayers. Imaging many samples over large areas indicates that this morphology persists over the entirety of the coated substrate area and confirms that the LB approach yields higher quality films [37, 38] compared to those deposited by spin [16] or dip-coating [18]. These latter methods typically result in a more random deposition, requiring more material to cover the same area. On the other hand, single layer films produced by our method leave about $5 \%$ of the area exposed compared to CVD grown films, the latter which may possess fewer film defects but 
remain more challenging and costly to produce. It was challenging to verify the film morphology and coverage on PET substrates directly because the surface roughness is larger than the expected film thickness and the secondary electron contrast difference between PET and RGO is poor. However, the fact that we could achieve similar morphologies on both hydrophilic (mica) and hydrophobic (HOPG) substrates suggests that our film coverage and thickness should be very similar on PET.

The optical transparency of the $60 \mathrm{ohms} / \mathrm{sq}$ AgNW films (not including the PET substrate) at a wavelength of $550 \mathrm{~nm}$ were $94 \%$. The transmittance of the AgNW electrode dropped from $94 \% 91 \%$ after the deposition of the RGO layer. The observed $3 \%$ transmittance decrease is consistent with the transmittance value of a single layer of graphene in the visible range which is in direct agreement with our AFM and SEM analysis of the graphene film discussed above [39]. The RGO sheets are electrically conductive, however, their electrical conductivity is significantly less than the conductivity of the nanowires [40], and the contact resistance between two overlapping RGO sheets is high. Thus, the overall sheet resistance of the AgNW electrodes did not decrease significantly after adding the RGO layer (only by $0 \% 10 \%$ ).

\subsection{Annealing study}

We first confirmed prior studies of the literature showing the effectiveness of RGO in protecting the nanowire films against degradation when annealed in a furnace. Both the AgNW and AgNW-RGO electrodes were annealed at $70{ }^{\circ} \mathrm{C}$ in air for 14 days. After 6 days, the sheet resistance of the AgNW electrode increased by a factor of 180 compared to the initial sheet resistance of $60 \mathrm{ohms} / \mathrm{sq}$, whereas the sheet resistance of the AgNW-RGO electrode increased only by a factor of 1.5 after 14 days. This indicates that the RGO layer is quite successful in slowing down silver nanowire electrode degradation under annealing conditions, as concluded by many others. However, testing its effectiveness under current flow is essential to mimic the stress faced by the AgNW electrode during use in a solar cell or OLED.

\subsection{Surface temperature distribution under current flow}

A $20 \mathrm{~mA}$ DC current was passed across the electrodes per centimetre length of the current collectors $\left(20 \mathrm{~mA} \mathrm{~cm}^{1}\right)$. Figure 2 shows the evolution of the surface temperature profiles obtained using thermal imaging for $60 \mathrm{ohms} / \mathrm{sq}$ ITO, $\mathrm{AgNW}$, and AgNW-RGO electrodes. Temperature images in figures 2(a), (d), (g) are shown $10 \mathrm{~s}$ after the current flow was applied whereas images in figures $2(\mathrm{~b}),(\mathrm{e}),(\mathrm{h})$ are recorded after the temperature has stabilized (60 s). In these images, the current flows from top to bottom, as indicated schematically in figure 2(a). As can be seen, the temperature appears uniform over the ITO electrode. However, local temperature variations greater than $20^{\circ} \mathrm{C}$ are evidenced across the AgNW electrode (figures 2(d) (f)). The presence of such hot spots in nanowire electrodes has also been reported by others [41, 42] and may be due to the non-uniform density distribution of the nanowires across the electrode. In bottleneck regions where there is a sparser nanowire concentration, individual nanowires must sustain high current densities and thus local Joule heating will be significantly higher than in denser nanowire regions. The hotspots observed in the unpassivated nanowire electrode exist along a line across the electrode parallel to the current collectors. We hypothesize that there are regions of sparse nanowires along this line and thus much of the current cannot re-route through denser locations. Thus Joule heating, equal to $I^{2} R$ where $I$ is current and $R$ is resistance, is high along this line. The existence of hot spots accelerates both corrosion and Rayleigh instability in these regions leading to nanowire breakdown. When nanowires break down, more current is forced through other available pathways, causing those regions to then locally increase in temperature. This situation can be observed in figures 2(d) (f). A hotspot present after $10 \mathrm{~s}$, which figure 2(f) indicates is at the highest temperature on the electrode, no longer exists after $60 \mathrm{~s}$. This suggests that some nanowires failed rapidly after current was applied due to this high temperature. As can be observed, it cooled down once current could no longer flow through that pathway, but consequently, the regions around the failed nanowires became hotter. This in turn accelerates nanowire degradation at these points until ultimately, as we observed when higher currents were applied to accelerate failure times, an open circuit exists in a line extending across the electrode.

It is important to note that the smallest detected area (pixel resolution) of the thermal imaging camera is $250 \mu \mathrm{m} \times 250 \mu \mathrm{m}$ which corresponds to an area with more than one hundred nanowires. Thus, the temperature of individual nanowires is not directly detectable, and we get an average temperature over the pixel area. In the modelling results reported later, we will see that the temperature variations on the nanoscale are far higher than ones observed on the microscale in thermal maps such as these.

In figures 2(g) (i) it is evidenced that the RGO passivation layer smooths out the temperature variation over the AgNW electrode such that the resulting temperature uniformity appears comparable to that of the ITO electrode. This is likely because of the high thermal conductivity of the RGO (above $1000 \mathrm{~W} \mathrm{~m}^{1} \mathrm{~K}^{1}$ ) [43] compared to air $\left(0.024 \mathrm{~W} \mathrm{~m}^{1} \mathrm{~K}^{1}\right)$ [44] and the plastic substrate $\left(0.20 \mathrm{~W} \mathrm{~m}^{1} \mathrm{~K}^{1}\right)$ [45] which assists in the lateral distribution of heat over the surface of the electrode. The absence of hot spots is one of the reasons why we will later see that the RGO extends the lifetime of AgNW electrodes under current flow. Also, because the RGO distributes the heat more evenly across the surface, it effectively provides a larger surface area from which the heat can dissipate and thereby slightly lowers the average surface temperature of the electrode.

\subsection{Electrode failure under current flow}

Figure 3(a) shows the average surface temperature (measured with a thermocouple that was attached to the bottom of the substrate) and sheet resistance evolution for an unpassivated $60 \mathrm{ohms} / \mathrm{sq} \mathrm{AgNW}$ electrode under $20 \mathrm{~mA} \mathrm{~cm}^{1}$ of linear 


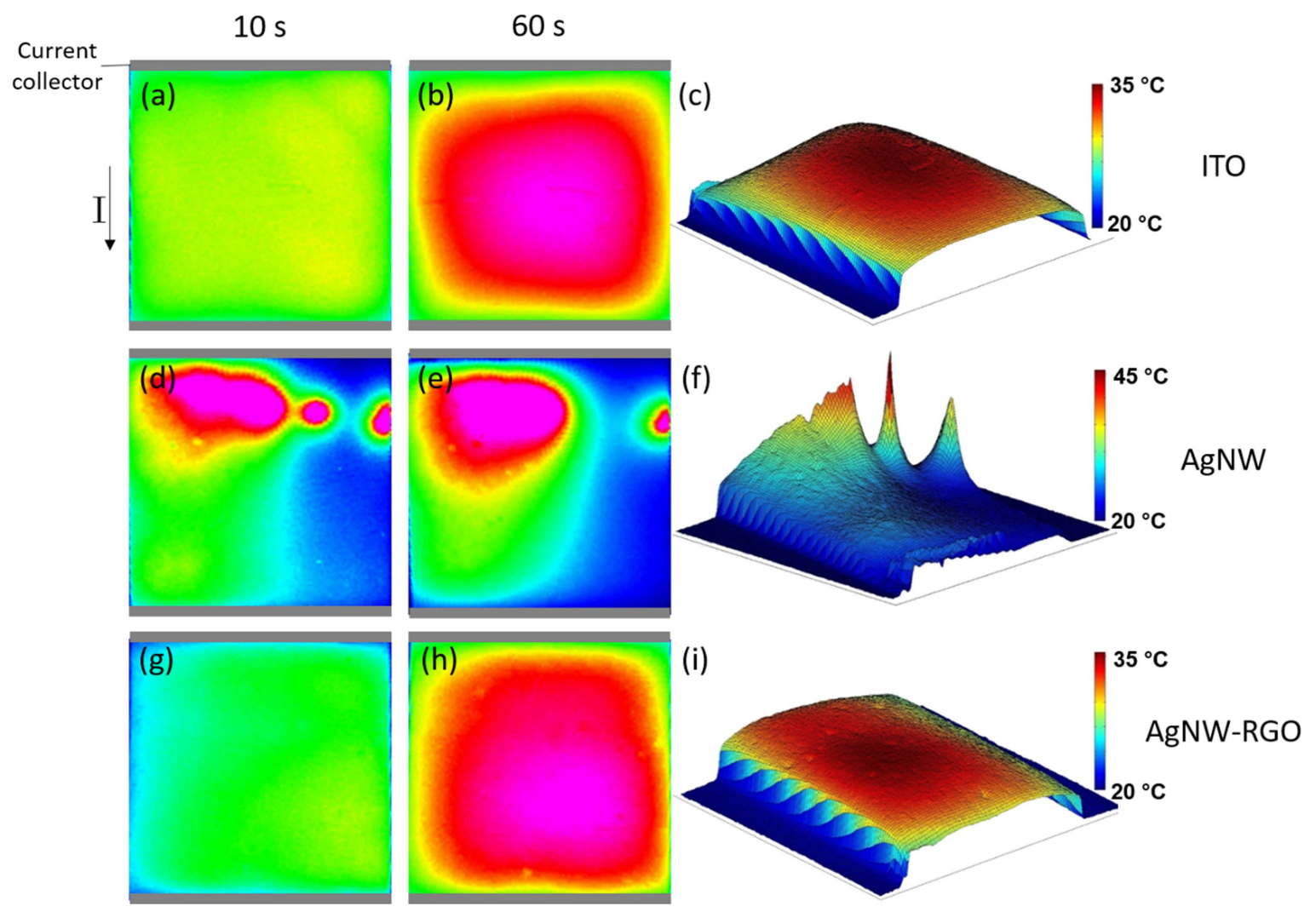

Figure 2. Temperature profiles of $2 \mathrm{~cm} \times 2 \mathrm{~cm}$ electrodes, measured using a thermal imaging camera, when $20 \mathrm{~mA} \mathrm{~cm}^{-1}$ of current is applied from top to bottom. (a), (b) surface temperature of ITO, (c) 3D temperature profile of ITO after $60 \mathrm{~s}$. (d), (e) Surface temperature of the AgNW electrode, (f) 3D temperature profile of the AgNW electrode after $10 \mathrm{~s}$. (g), (h) Surface temperature of the AgNW RGO electrode, (i) $3 \mathrm{D}$ temperature profile of the AgNW RGO electrode after $60 \mathrm{~s}$.

current density. The electrode failed after $120 \mathrm{~h}$ (5 days), where failure is defined as when the sheet resistance exceeded $1500 \mathrm{ohms} / \mathrm{sq}$. SEM images of nanowires after electrode failure are shown in figures $3(\mathrm{~b})$ and (c), illustrating the two different types of nanowire breakdown that were observed. In figure 3(b), the presence of nanoparticles on the nanowires indicate nanowire corrosion [5] which eventually lead to electrical discontinuities. Other nanowires (figure 3(c)) are completely melted in addition to damaging the plastic substrate underneath, implying a very high localized temperature. We suggest that these latter nanowires were subjected to very high levels of current and thus heated up and failed quickly, like those associated with the disappearing hotspot in figures 2(d) and (e), whereas the corroded nanowires were subject to lower current densities over a longer time period. These issues will be further discussed when the modelling of current levels and temperatures of individual nanowires is presented.

Figure 4(a) shows the average temperature and sheet resistance of the AgNW-RGO electrodes under a linear current of $20 \mathrm{~mA} \mathrm{~cm}{ }^{1}$. The RGO passivation layer extended the lifetime of the $\mathrm{AgNW}$ electrode to $285 \mathrm{~h}$ ( $\sim 12$ days). There are three possible mechanisms by which RGO passivates the electrodes: (1) nanowire corrosion is slowed since the RGO impedes air from reaching the nanowires. This is supported by the observed increase in lifetime upon extended annealing at $70{ }^{\circ} \mathrm{C}$ with no current flow; (2) the RGO prevents hotspots (as discussed above) where the fastest degradation occurs; and (3) the RGO increases the morphological stability of the nanowires and thus increases the temperature at which the nanowires break-up [46]. Indeed, SEM images taken after electrode failure show that the RGO-passivated electrodes do not fail due to NW melting or corrosion (figure 4(b)), which is the case for unpassivated electrodes. Rather, the electrode instead breaks down due to deformation and melting of the PET substrate on a micron length-scale (figures 4(c) and (d)). The substrate deformation causes breakage in the nanowires and thus electrical discontinuities.

A melted substrate indicates that the local temperature of the nanowires exceeds the melting point of PET $\left(\sim 250^{\circ} \mathrm{C}\right)$ during current flow. The silver nanowires, normally unstable at this temperature [47], remain due to the RGO passivation. Although the thermal imaging data indicated the RGO-passivated electrodes had an average surface temperature of $32{ }^{\circ} \mathrm{C}$ under this same current flow (figure 2(i)), the local temperature of some nanowires are much higher. These results demonstrate the inadequacy of annealing a nanowire electrode to assess stability and lifetimes, which is the method used by many others and done above. The Joule heating that would take place in an electrode during solar cell operation, which causes very high localized temperatures, is not incurred. As measured above, the resistance of the RGO-passivated electrode annealed in a furnace at $70{ }^{\circ} \mathrm{C}$ only increased by 1.5 times after 12 days, whereas under a current flow causing 

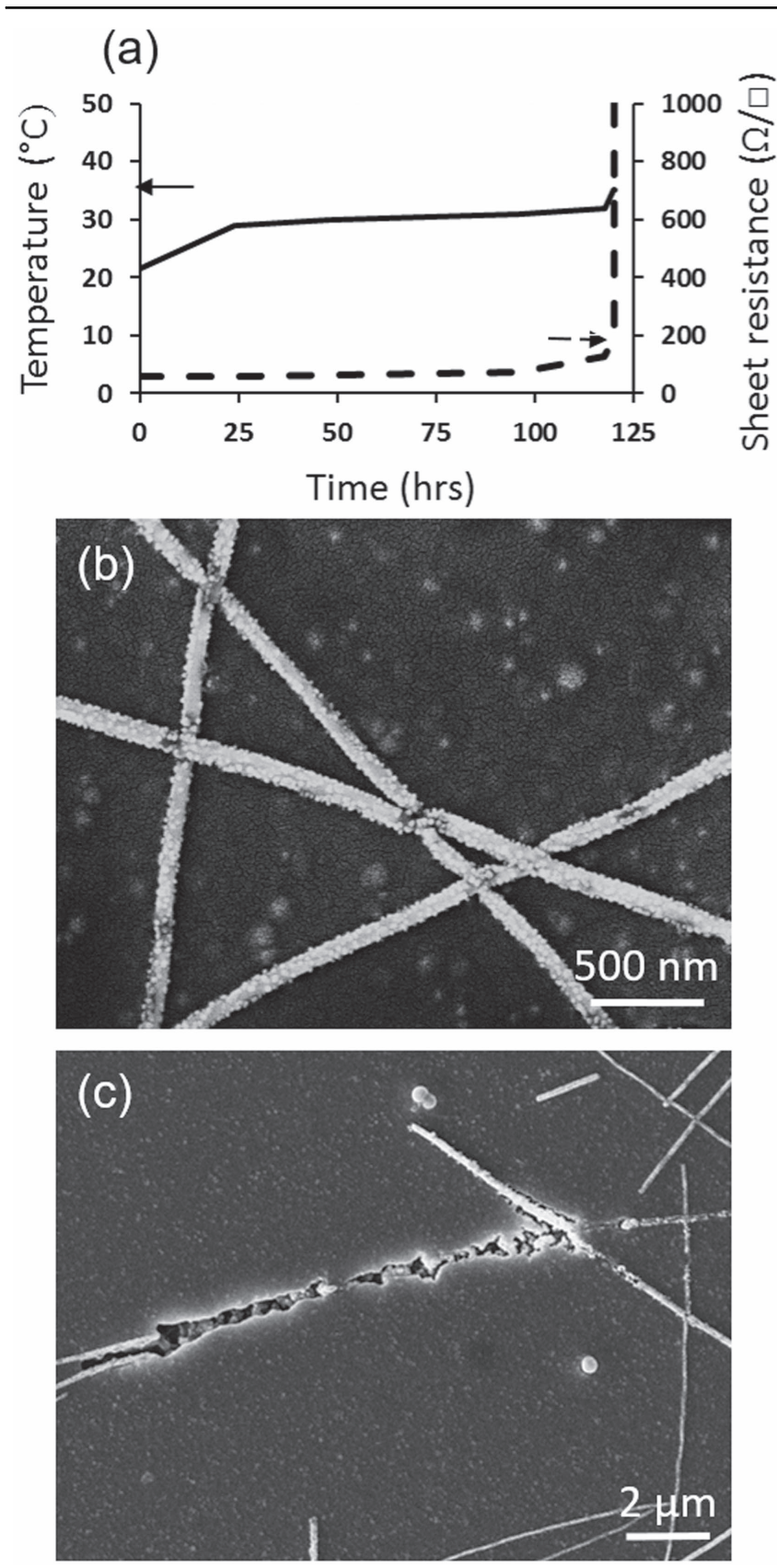

Figure 3. (a) Temperature and sheet resistance of a AgNW electrode over time under a linear current density of $20 \mathrm{~mA} \mathrm{~cm}^{-1}$. (b), (c) SEM images of nanowires after electrode failure. The nanowires in (b) show evidence of accelerated corrosion leading to disconti nuities, and in (c) a nanowire along with with part of the PET substrate underneath has melted.

an average surface temperature of $32{ }^{\circ} \mathrm{C}$, the resistance increased by more than 25 times.

\subsection{Modelling of current in a nanowire network}

As evidenced by SEM imaging, electrode failure occurs at the level of individual nanowires. However, measuring the local temperature and the levels of electric current flowing through individual nanowires in an electrode is experimentally prohibitive. Therefore, modelling was employed to better understand the extent of Joule heating in an electrode and the mechanisms of failure.

The silver network modelled in MATLAB is shown in figure 5(a). Figure 5(b) tabulates the typical current densities in individual nanowire segments (segments span between two red dots in figure 5(a), where red dots are the locations of nanowire junctions and nanowire endpoints) when $20 \mathrm{mAcm}^{1}$ is applied across the metal end bars. This level of current does not lead to problematic current densities in continuous films, however, in the case of nanowire electrodes, as previously discussed, high current densities occur when the current is forced through $90 \mathrm{~nm}$ thick nanowire pathways. The average current density in the nanowires is calculated to be $1.2 \pm$ $0.1 \times 10^{5} \mathrm{~A} \mathrm{~cm}^{2}$. As a comparison, in the case of a $130 \mathrm{~nm}$ thick film of ITO corresponding to the approximate thickness to achieve $60 \mathrm{ohms} / \mathrm{sq}$, the current density would be $1.5 \times 10^{3} \mathrm{~A} \mathrm{~cm}^{2}$, two orders of magnitude less than the one observed in the nanowires. $0.5 \pm 0.1 \%$ (error based on running the simulation with a different random network 5 times) of the nanowires in the electrode may carry a current density superior to $5.5 \times 10^{5} \mathrm{~A} \mathrm{~cm}^{2}$ and are expected to break down relatively fast due to Joule heating (to be discussed in the subsequent section).

\subsection{Joule heating modelling of nanowires in electrodes}

Figure 6 shows the results of the COMSOL Joule heating simulations and the steady state temperatures reached by an individual AgNW, a AgNW passivated with RGO, and ITO. It is worth mentioning that at steady state the whole system is at a similar temperature and thus the colouring indicates only very minor differences in temperature. The colours however are useful to show how heat is dissipated in the system. A single AgNW carrying the average current previously calculated in the section above $\left(1.2 \times 10^{5} \mathrm{~A} \mathrm{~cm}^{2}\right)$ reached $33^{\circ} \mathrm{C}$ (figure 6(a)) and a RGO-passivated $\mathrm{AgNW}$ reached $30^{\circ} \mathrm{C}$ (figure 6(b)). The RGO film helps spread out the heat and reduces the surface temperature by $8 \% 10 \%$. Our simulated results match closely to experiment: the calculated average surface temperature of the AgNW electrode under $20 \mathrm{~mA} \mathrm{~cm}^{1}$ of current, recorded by the IR camera, was found to be $33 \pm 2{ }^{\circ} \mathrm{C}$, and the measured average temperature of the AgNW-RGO (figure 2(i)) was $32 \pm 2{ }^{\circ} \mathrm{C}$. In the case of a $60 \mathrm{ohms} / \mathrm{sq}, 130 \mathrm{~nm}$ thick ITO film on a PET substrate under the calculated ITO average current density of $1.5 \times 10^{3} \mathrm{~A} \mathrm{~cm}^{2}$ (figure 6(c)), the steady state temperature was calculated to be $32^{\circ} \mathrm{C}$, also close to the $34 \pm 2{ }^{\circ} \mathrm{C}$ average temperature recorded by the IR camera (figure 2(c)).

Although the temperature of a nanowire conducting the average current density is $33{ }^{\circ} \mathrm{C}$, there are some individual nanowire segments in the whole metallic network that can become much hotter. Figure 6(d) shows the simulated temperature of silver nanowires on a PET substrate at different current densities. As calculated in the previous section, $0.5 \%$ of the nanowire segments in a $60 \mathrm{ohms} / \mathrm{sq} \mathrm{AgNW}$ electrode under $20 \mathrm{~mA} \mathrm{~cm}{ }^{1}$ current flow are carrying a current density higher than $5.5 \times 10^{5} \mathrm{~A} \mathrm{~cm}^{2}$. Figure 6(d) shows that the temperature of unpassivated NWs carrying such levels of 

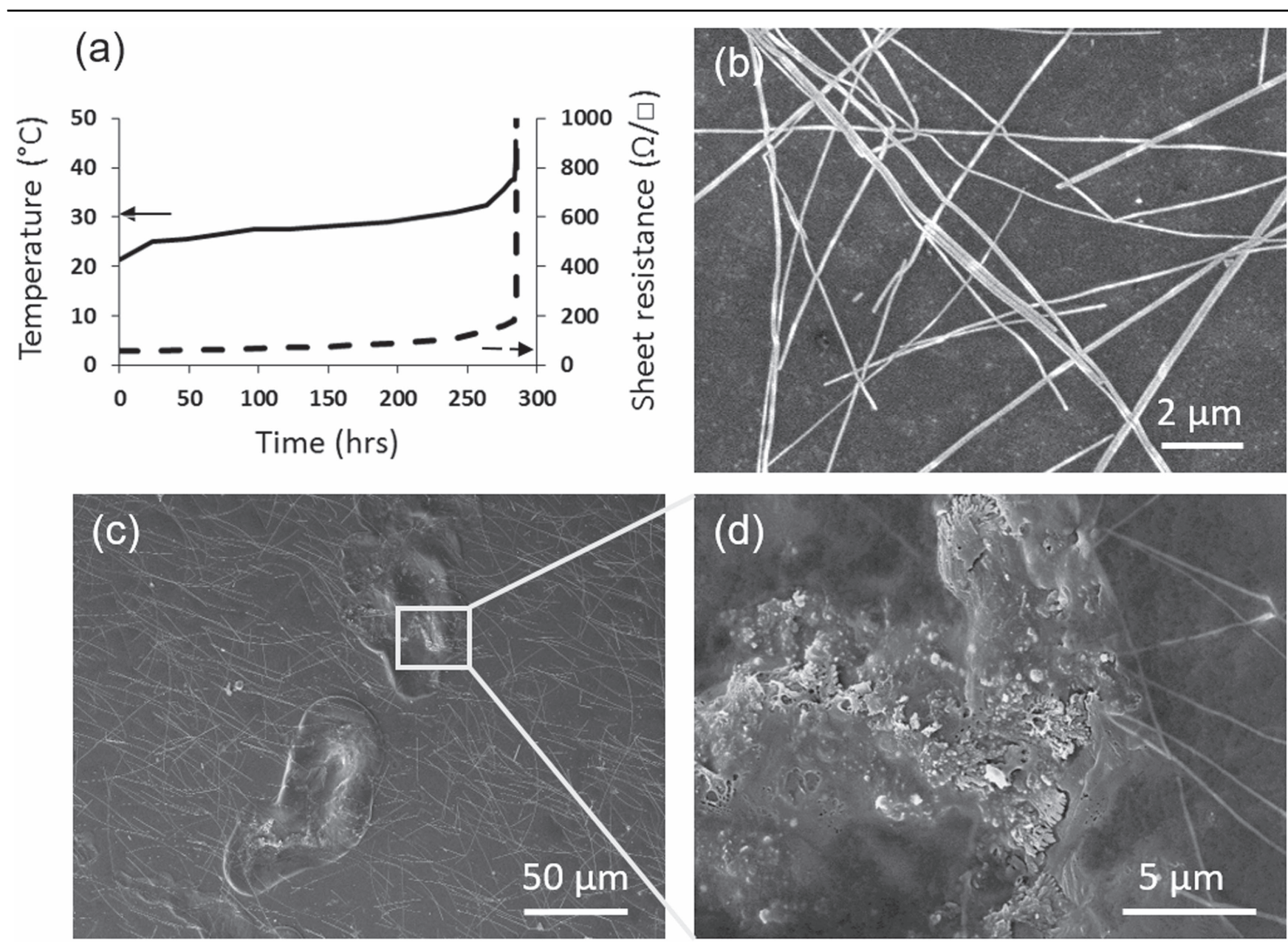

Figure 4. (a) Temperature and sheet resistance versus time of a AgNW RGO electrode under a linear current density of $20 \mathrm{~mA} \mathrm{~cm}{ }^{-1}$. SEM images after failure of (b) a non melted region and (c), (d) localized substrate melting.

current exceed $250{ }^{\circ} \mathrm{C}$, a temperature above the melting point of PET. Importantly, at such a temperature unpassivated $90 \mathrm{~nm}$ diameter AgNWs are not thermally stable either [47]. Such nanowires would fail quickly after the current is applied. These nanowire segments are hotter than the hottest spot evidenced in the IR images because, as mentioned above, the camera measures an average temperature of several hundred nanowires. However, the existence of these very hot nanowires still corroborates with our experimental results. The IR data in figures $2(\mathrm{~d})$ and (e) insinuate that there is a rapid failure of some nanowires, and the SEM image in figure 3(c) indicates the melting of both the nanowire and a localized portion of the PET substrate. Once these high current carrying nanowires break and are no longer conductive, the current is redistributed amongst the other nanowires.

The existence of localized nanoscale hotspots of high temperatures is in stark contrast to ITO electrodes where the current is distributed evenly throughout the film. In an ITO film carrying the same linear current density as the nanowire electrode, all points over the surface are expected to be close to $32{ }^{\circ} \mathrm{C}$. The existence of a non-uniform temperature distribution and localized hotspots reaching $250^{\circ} \mathrm{C}$ is a unique issue for nanowire electrodes.
Because RGO is not as electrically conductive as the nanowire network, it does not significantly lower the current densities in the nanowires and therefore Joule heating still occurs. Although it initially avoids larger microscale hotspots and slightly lowers the average surface temperature by spreading heat more uniformly over the surface, we can see in figure 6(d) that the temperatures of individual nanowires in the AgNW-RGO case can still be very high. And because a single layer of RGO does not thoroughly prevent nanowire degradation, as evidenced by the slight resistance increase during annealing (1.5 times after 14 days) and by the observation of some nanowire corrosion in the SEM after extended current flow, the current densities in the nanowires increase over time. As a consequence, this leads to more and more Joule heating until ultimately the PET substrate melts over larger microscale areas (rather than melting only underneath the nanowires in the case of unpassivated electrodes, since the RGO distributes the heat beyond the location of an individual nanowire). This causes the nanowire networks to distort and fail.

In addition to concerns about electrode failure, our models show that the temperature of $5.5 \pm 0.5 \%$ of the unpassivated nanowires and $5.0 \pm 0.5 \%$ of the RGO-passivated nanowires may exceed $100^{\circ} \mathrm{C}$, which could adversely 
(a)

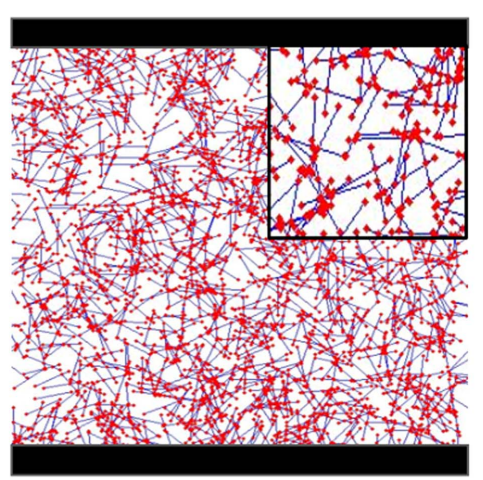

(b)

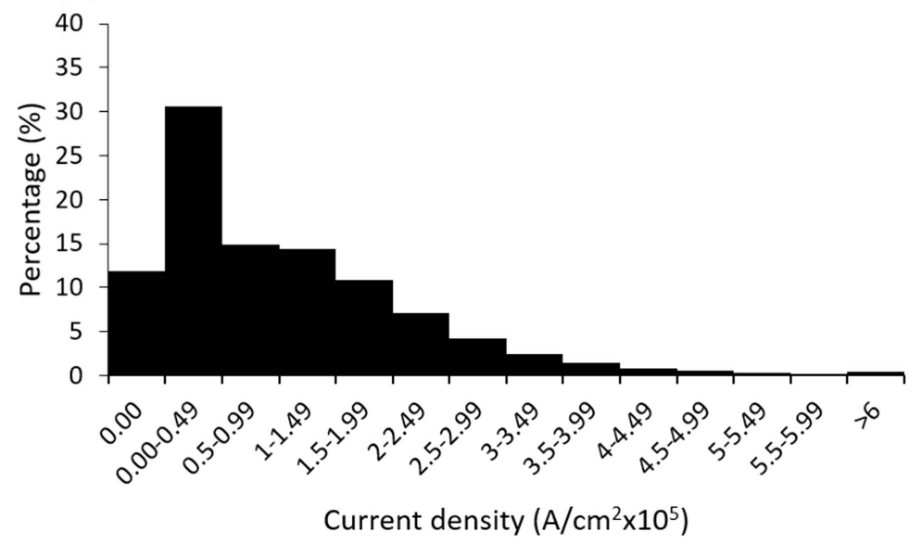

Figure 5. (a) Silver nanowire electrode modelled as a random network of interconnected conducting sticks with a sheet resistance of $60 \mathrm{ohms} / \mathrm{sq}$ (red dots show nanowire junctions and sticks endpoints). (b) The distribution of current densities in individual nanowire segments (i.e. between two red dots) of the nanowire electrode.

(a)

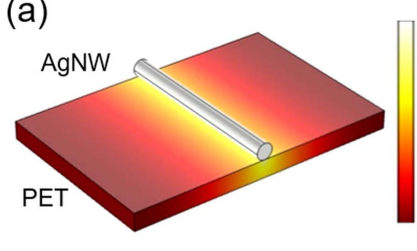

(b) $33^{\circ} \mathrm{C}$

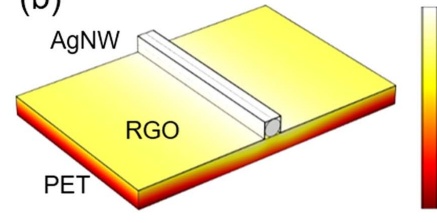

(c)

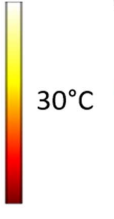

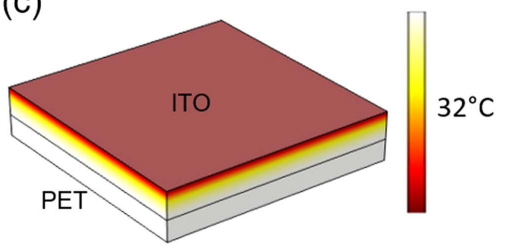

(d)

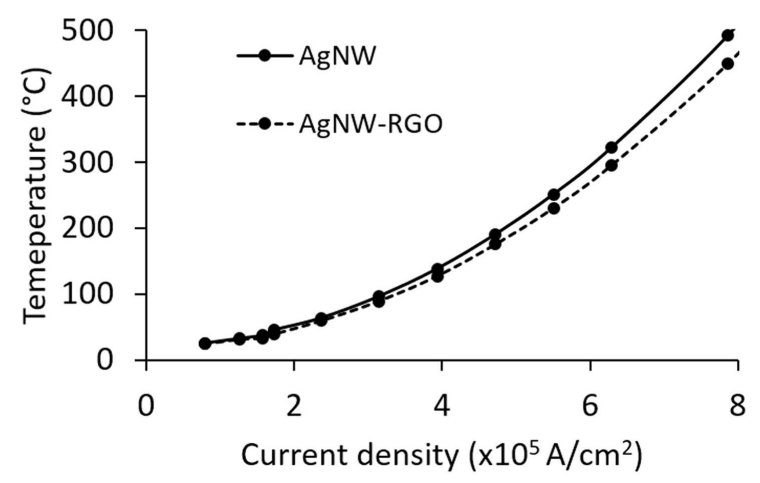

Figure 6. Steady state thermal profile of a (a) AgNW, (b) RGO passivated AgNW, and (c) $130 \mathrm{~nm}$ thick film of ITO on PET when they are carrying the average current density encountered when $20 \mathrm{~mA} \mathrm{~cm}^{-1}$ is passed across the electrode. (d) Calculated steady state temperature of individual unpassivated and passivated silver nanowires on PET substrates under different current densities.

affect other materials on top of the electrode in a device. Because organic materials are not stable at high temperatures, the maximum operating temperature for organic solar cells and LEDs is commonly below $100{ }^{\circ} \mathrm{C}$ [48 51]. This is lower than the melting point of PET and thus these devices require a more stringent thermal budget. This is another reason why Joule heating in nanowire electrodes is a major concern that needs to be addressed.

\subsection{Managing Joule heating}

Our results show that a passivation layer alone is not sufficient to manage the Joule heating problem in silver nanowire transparent electrodes. In order to reduce Joule heating, the current density in individual nanowires needs to be reduced.
One way to achieve this is to increase the density of the nanowires in the electrode so that there are more current pathways. A $20 \mathrm{ohms} / \mathrm{sq} \mathrm{AgNW}$ electrode was modelled (figure 7(a)) and the current density distribution in the nanowire segments when $20 \mathrm{mAcm}^{1}$ of current is applied across the electrode is plotted in figure 7(b). The average current density in the nanowire segments is $0.6 \pm 0.1 \times 10^{5} \mathrm{~A} \mathrm{~cm}{ }^{2}$, which is half of the average current density expected in a $60 \mathrm{ohms} / \mathrm{sq}$ AgNW electrode.

The simulation results of Joule heating in the $20 \mathrm{ohms} / \mathrm{sq}$ electrodes are shown in figures 7(c) and (d). The steady state temperature of the AgNW and AgNW-RGO samples under the average current density reached to $27^{\circ} \mathrm{C}$ and $25^{\circ} \mathrm{C}$, respectively (figures 7 (c) and (d)), which are $6^{\circ}$ and $5^{\circ}$ lower than in the $60 \mathrm{ohms} / \mathrm{sq}$ case. In the AgNW and AgNW-RGO 
(a)

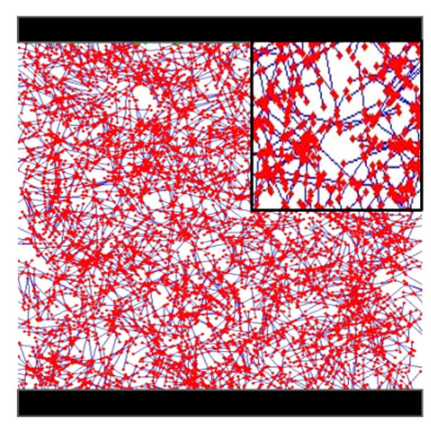

(c)

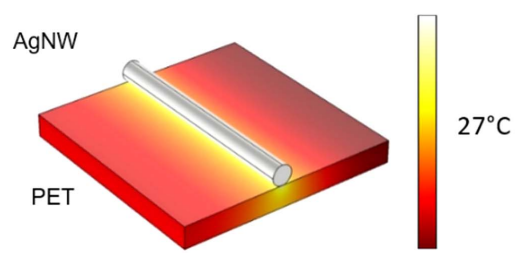

(b)

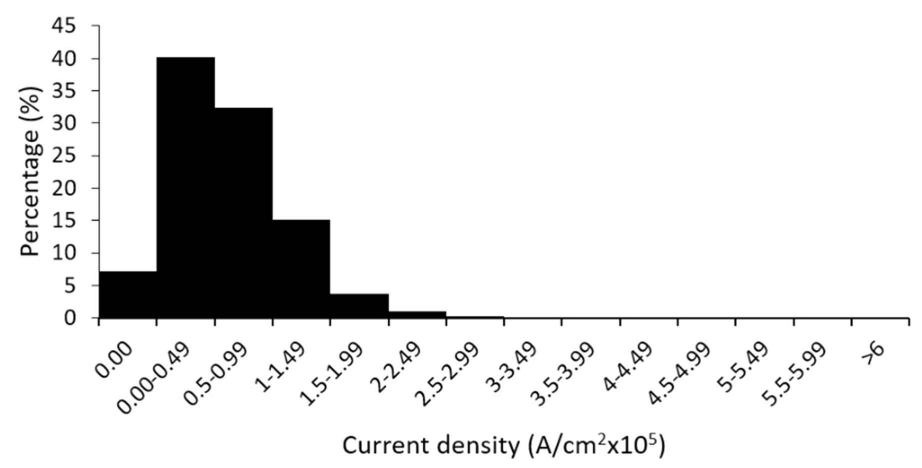

(d)

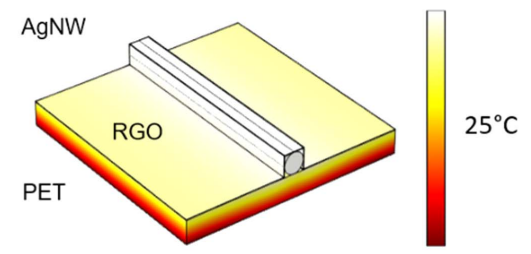

Figure 7. (a) Silver nanowire electrode model with a sheet resistance of $20 \mathrm{ohms} / \mathrm{sq}$ (red dots show nanowire junctions and endpoints). (b) The distribution of current densities in individual nanowire segments (i.e. between two red dots) of the 20 ohms/sq nanowire electrode. Steady state thermal profile of a (c) AgNW and (d) RGO passivated AgNW under the average current density of $0.6 \times 10^{5} \mathrm{~A} \mathrm{~cm}{ }^{-2}$.

electrodes, less than $0.006 \pm 0.003 \%$ and $0.002 \pm 0.001 \%$ of the nanowires, respectively, carry a current that results in a temperature above $250{ }^{\circ} \mathrm{C}$. In addition, only $0.45 \pm 0.15 \%$ and $0.15 \pm 0.1 \%$ of the nanowires get hotter than $100{ }^{\circ} \mathrm{C}$ in the $\mathrm{AgNW}$ and AgNW-RGO electrodes, respectively.

Experimentally, $20 \mathrm{mAcm}^{1}$ was passed across a $20 \mathrm{ohms} / \mathrm{sq}$ unpassivated $\mathrm{AgNW}$ electrode. The surface temperature was $26{ }^{\circ} \mathrm{C}, 6{ }^{\circ} \mathrm{C}$ lower than what was measured for the $60 \mathrm{ohms} / \mathrm{sq}$ case (figure 3(a)). The electrode failed after 25 days of sustained current compared to failure after 5 days for the $60 \mathrm{ohms} / \mathrm{sq}$ electrode. The effect of elevated currents was also assessed; under a sustained current of $50 \mathrm{~mA} \mathrm{~cm}{ }^{1}$, a $20 \mathrm{ohms} / \mathrm{sq} \mathrm{AgNW}$ electrode failed after 7 days. Overall, the effect of Joule heating is reduced in NW electrodes with lower sheet resistance and the lifetime is thus increased. Increasing the nanowire density in the electrodes has drawbacks however; the electrode transparency would decrease and the material cost would increase.

A second way to reduce the current density in individual nanowires is to, of course, have less current flowing across the electrode. In a solar cell this is achieved by making the active area smaller, or in other words, making the electrical contacts (current collectors) closer together, to lower the maximum current levels which occur near a contact. This, however, reduces the efficiency of the solar cells by increasing the surface area covered by the metal bars.

Thirdly, because excessive Joule heating leading to NW breakdown is most likely to occur in areas of sparse NWs, the nanowire networks should be made as uniformly dense as possible. However, in a random film there will always be variations in local density. Assembling the NWs into a regularly spaced grid, as has been achieved with some success using NW alignment techniques [52, 53], or depositing a

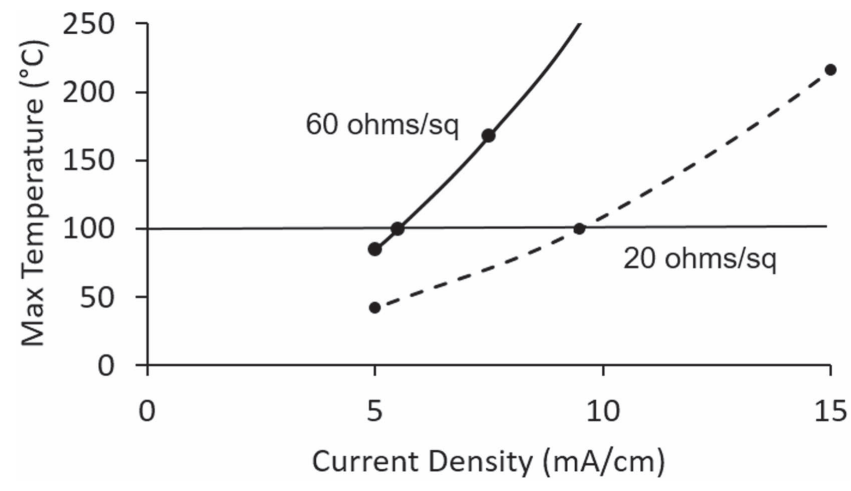

Figure 8. Maximum nanowire temperature in electrodes with sheet resistances of 20 and $60 \mathrm{ohms} / \mathrm{sq}$ as a function of linear current density between the current collectors.

Ag grid using patterning techniques [54, 55], would result in a more uniform current distribution and thus avoid hot spots. This would extend electrode lifetimes though at a cost of a more complex deposition process.

If the temperature of a $\mathrm{AgNW}$ electrode needs to remain below a certain value during operation for a given device (e.g. an organic device which cannot tolerate temperatures above $100^{\circ} \mathrm{C}$ ), our model and simulations can calculate the maximum linear current density that can be conducted. Figure 8 shows the temperature of the hottest nanowire in $300 \mu \mathrm{m} \times 300 \mu \mathrm{m}$ electrodes with sheet resistances of 20 and $60 \mathrm{ohms} / \mathrm{sq}$. If the thermal budget of a device is $100{ }^{\circ} \mathrm{C}$, for example, the maximum linear current density that should be conducted across the $60 \mathrm{ohms} / \mathrm{sq}$ electrode is $5.5 \mathrm{~mA} \mathrm{~cm}{ }^{1}$. This number increases to $9.5 \mathrm{~mA} \mathrm{~cm}^{1}$ for an electrode with a sheet resistance of $20 \mathrm{ohms} / \mathrm{sq}$. 


\section{Conclusion}

This study demonstrates that the Joule heating taking place in silver nanowire electrodes when they continuously conduct current is a much more serious problem than in conventional transparent homogeneous conductive electrodes like ITO. When a 60 ohms/sq AgNW electrode conducts current at the levels incurred near the metal contacts of organic solar cells, the resulting Joule heating leads to electrode failure in 5 days. An RGO passivation layer extends the electrode lifetime, but only to 12 days since it cannot prevent the Joule heating that ultimately leads to failure due to localized melting of the plastic substrate. Modelling indicates that the temperature of some individual nanowires under current flow reach much higher temperatures than the average surface temperature recorded. This explains the observation of substrate melting and the faster failure times as compared to electrodes whose lifetimes are assessed by annealing rather than current flow. If silver nanowire electrodes are used in solar cells, OLEDs, or transparent heaters, steps must be taken to reduce the Joule heating problem to extend both the lifetime of the electrode and the rest of the device as well. Lowering the electrode sheet resistance or lowering the current flowed across the electrode would both be effective strategies.

\section{Acknowledgments}

HHK, LX, MAP, and IAG gratefully acknowledge funding provided by the Natural Science and Engineering Research Council (NSERC) of Canada. MR and CP acknowledge support of the CNRS National Science Foundation under the grant «IMAG'IN » No. 58057.

\section{References}

[1] Ye S, Rathmell A R, Chen Z, Stewart I E and Wiley B J 2014 Metal nanowire networks: the next generation of transparent conductors Adv. Mater. 26667087

[2] Sannicolo T, Lagrange M, Cabos A, Celle C, Simonato J P and Bellet D 2016 Metallic nanowire based transparent electrodes for next generation flexible devices: a review Small 12605275

[3] Chen D, Liang J and Pei Q 2016 Flexible and stretchable electrodes for next generation polymer electronics: a review Sci. China Chem. 5965971

[4] Khaligh H H, Liew K, Han Y, Abukhdeir N M and Goldthorpe I A 2015 Silver nanowire transparent electrodes for liquid crystal based smart windows Sol. Energy Mater. Sol. Cells 13233741

[5] Khaligh H H and Goldthorpe I A 2013 Failure of silver nanowire transparent electrodes under current flow Nanoscale Res. Lett. 8235

[6] Elechiguerra J L, Larios Lopez L, Liu C, Garcia Gutierrez D, Camacho Bragado A and Yacaman M J 2005 Corrosion at the nanoscale: the case of silver nanowires and nanoparticles Chem. Mater. 17604252

[7] Franey J P, Kammlott G W and Graedel T E 1985 The corrosion of silver by atmospheric sulfurous gases Corros. Sci. 2513343
[8] Langley D P, Lagrange M, Giusti G, Jiménez C, Bréchet Y, Nguyen N D and Bellet D 2014 Metallic nanowire networks: effects of thermal annealing on electrical resistance Nanoscale 61353543

[9] Garnett E C, Cai W, Cha J J, Mahmood F, Connor S T, Greyson Christoforo M, Cui Y, McGehee M D and Brongersma M L 2012 Self limited plasmonic welding of silver nanowire junctions Nat. Mater. 112419

[10] Karim S, Toimil Molares M E, Balogh A G, Ensinger W, Cornelius T W, Khan E U and Neumann R 2006 Morphological evolution of $\mathrm{Au}$ nanowires controlled by Rayleigh instability Nanotechnology 175954

[11] Ramasamy P, Seo D M, Kim S H and Kim J 2012 Effects of $\mathrm{TiO}_{2}$ shells on optical and thermal properties of silver nanowires J. Mater. Chem. 2211651

[12] Morgenstern F S F, Kabra D, Massip S, Brenner T J K, Lyons P E, Coleman J N and Friend R H 2011 Ag nanowire films coated with $\mathrm{ZnO}$ nanoparticles as a transparent electrode for solar cells Appl. Phys. Lett. 99183307

[13] Deng B et al 2015 Roll to roll encapsulation of metal nanowires between graphene and plastic substrate for high performance flexible transparent electrodes Nano Lett. 15 420613

[14] Zhang X, Yan X, Chen J and Zhao J 2014 Large size graphene microsheets as a protective layer for transparent conductive silver nanowire film heaters Carbon 6943743

[15] Nair R R, Wu H A, Jayaram P N, Grigorieva I V and Geim A K 2012 Unimpeded permeation of water through helium leak tight graphene based membranes Science $\mathbf{3 3 5}$ 4424

[16] Liu B T and Kuo H L 2013 Graphene/silver nanowire sandwich structures for transparent conductive films Carbon 633906

[17] Naito K, Yoshinaga N, Tsutsumi E and Akasaka Y 2013 Transparent conducting film composed of graphene and silver nanowire stacked layers Synth. Met. 175426

[18] Chen R, Das S R, Jeong C, Khan M R, Janes D B and Alam M A 2013 Co percolating graphene wrapped silver nanowire network for high performance, highly stable, transparent conducting electrodes $A d v$. Funct. Mater. 23 51508

[19] Yang S B, Choi H, Lee D S, Choi C G, Choi S Y and Kim I D 2015 Improved optical sintering efficiency at the contacts of silver nanowires encapsulated by a graphene layer Small $\mathbf{1 1}$ 1293300

[20] Ahn Y, Jeong Y and Lee Y 2012 Improved thermal oxidation stability of solution processable silver nanowire transparent electrode by reduced graphene oxide ACS Appl. Mater. Interfaces 464104

[21] Lee D, Lee H, Ahn Y and Lee Y 2015 High performance flexible transparent conductive film based on graphene/ $\mathrm{AgNW} /$ graphene sandwich structure Carbon 8143946

[22] Hu L, Kim H S, Lee J Y, Peumans P and Cui Y 2010 Scalable coating and properties of transparent, flexible, silver nanowire electrodes ACS Nano 4295563

[23] Khaligh H H 2013 Silver nanowire transparent electrodes: fabrication, characterization, and device integration $M S C$ Thesis University of Waterloo

[24] Khaligh H H and Goldthorpe I A 2014 Hot rolling nanowire transparent electrodes for surface roughness minimization Nanoscale Res. Lett. 9310

[25] Jørgensen M et al 2013 The state of organic solar cells a meta analysis Sol. Energy Mater. Sol. Cells 1198493

[26] Green M A, Emery K, Hishikawa Y, Warta W and Dunlop E D 2015 Solar cell efficiency tables (Version 45) Prog. Photovolt., Res. Appl. 2319

[27] Yu Z, Zhang Q, Li L, Chen Q, Niu X, Liu J and Pei Q 2011 Highly flexible silver nanowire electrodes for shape memory polymer light emitting diodes Adv. Mater. 236648 
[28] Niggemann M, Zimmermann B, Haschke J, Glatthaar M and Gombert A 2008 Organic solar cell modules for specific applications from energy autonomous systems to large area photovoltaics Thin Solid Films 51671817

[29] Yang J, Vak D, Clark N, Subbiah J, Wong W W H, Jones D J, Watkins S E and Wilson G 2013 Organic photovoltaic modules fabricated by an industrial gravure printing proofer Sol. Energy Mater. Sol. Cells 1094755

[30] Tsai P T, Yu K C, Chang C J, Horng S F and Meng H F 2015 Large area organic solar cells by accelerated blade coating Org. Electron. 2216672

[31] Marcano D C, Kosynkin D V, Berlin J M, Sinitskii A, Sun Z, Slesarev A, Alemany L B, Lu W and Tour J M 2010 Improved synthesis of graphene oxide ACS Nano 4480614

[32] Pron H and Bissieux C 2004 Focal plane array infrared cameras as research tools Quant. InfraRed Thermogr. J. 1 22940

[33] Romano M, Pradere C, Toutain J, Hany C and Batsale J C 2014 Quantitative thermal analysis of heat transfer in liquid liquid biphasic millifluidic droplet flows Quant. InfraRed Thermogr. J. 1113460

[34] Bellew A T, Manning H G, Gomes da Rocha C, Ferreira M S and Boland J J 2015 Resistance of single Ag nanowire junctions and their role in the conductivity of nanowire networks ACS Nano 9114229

[35] Bid A, Bora A and Raychaudhuri A K 2006 Temperature dependence of the resistance of metallic nanowires of diameter $\geqslant 15 \mathrm{~nm}$ : applicability of Bloch Grüneisen theorem Phys. Rev. B 7435426

[36] Cheng Z, Liu L, Xu S, Lu M and Wang X 2015 Temperature dependence of electrical and thermal conduction in single silver nanowire Sci. Rep. 510718

[37] Pope M A, Punckt C and Aksay I A 2011 Intrinsic capacitance and redox activity of functionalized graphene sheets $J$. Phys. Chem. C 1152032634

[38] Cote L J, Kim F and Huang J 2009 Langmuir Blodgett assembly of graphite oxide single layers J. Am. Chem. Soc. 13110439

[39] Li X, Zhu Y, Cai W, Borysiak M, Han B, Chen D, Piner R D, Colombo L and Ruoff R S 2009 Transfer of large area graphene films for high performance transparent conductive electrodes Nano Lett. 9435963

[40] Eda G, Fanchini G and Chhowalla M 2008 Large area ultrathin films of reduced graphene oxide as a transparent and flexible electronic material Nat. Nanotechnol. 32704

[41] Woo J S, Han J T, Jung S, Jang J I, Kim H Y, Jeong H J, Jeong S Y, Baeg K J and Lee G W 2014 Electrically robust metal nanowire network formation by in situ interconnection with single walled carbon nanotubes Sci. Rep. 44804

[42] Hong S, Lee H, Lee J, Kwon J, Han S, Suh Y D, Cho H, Shin J, Yeo J and Ko S H 2015 Highly stretchable and transparent metal nanowire heater for wearable electronics applications Adv. Mater. 27474451

[43] Kumar P, Shahzad F, Yu S, Hong S M, Kim Y H and Koo C M 2015 Large area reduced graphene oxide thin film with excellent thermal conductivity and electromagnetic interference shielding effectiveness Carbon 94 494500

[44] Montgomery R B 1947 Viscosity and thermal conductivity of air and diffusivity of water vapor in air J. Meteorol. 4 1936

[45] Lopes C M A and Felisberti M I 2004 Thermal conductivity of PET/(LDPE/AI) composites determined by MDSC Polym. Test. 2363743

[46] Kwan Y C G, Le Q L and Huan C H A 2016 Time to failure modeling of silver nanowire transparent conducting electrodes and effects of a reduced graphene oxide over layer Sol. Energy Mater. Sol. Cells 1441028

[47] Lee J Y, Connor S T, Cui Y and Peumans P 2008 Solution processed metal nanowire mesh transparent electrodes Nano Lett. 868992

[48] Sachs Quintana I T, Heumüller T, Mateker W R, Orozco D E, Cheacharoen R, Sweetnam S, Brabec C J and McGehee M D 2014 Electron barrier formation at the organic back contact interface is the first step in thermal degradation of polymer solar cells Adv. Funct. Mater. 24397885

[49] Katz E A, Faiman D, Tuladhar S M, Kroon J M, Wienk M M, Fromherz T, Padinger F, Brabec C J and Sariciftci N S 2001 Temperature dependence for the photovoltaic device parameters of polymer fullerene solar cells under operating conditions J. Appl. Phys. 90534350

[50] Bergemann K J, Krasny R and Forrest S R 2012 Thermal properties of organic light emitting diodes Org. Electron. 13 15658

[51] Thao T T, Trung T Q, Truong V V, Dinh N N, Thao T T, Trung T Q, Truong V V and Dinh N N 2015 Enhancement of power efficiency and stability of P3HT based organic solar cells under elevated operating temperatures by using a nanocomposite photoactive layer J. Nanomater. 2015 463565

[52] Duan S, Niu Q, Wei J, He J, Yin Y and Zhang Y 2015 Water bath assisted convective assembly of aligned silver nanowire films for transparent electrodes Phys. Chem. Chem. Phys. PCCP 17810612

[53] Whang D, Jin S, Wu Y and Lieber C M 2003 Large scale hierarchical organization of nanowire arrays for integrated nanosystems Nano Lett. 312559

[54] Sciacca B, van de Groep J, Polman A and Garnett E C 2016 Solution grown silver nanowire ordered arrays as transparent electrodes Adv. Mater. 289059

[55] Van de Groep J, Spinelli P and Polman A 2012 Transparent conducting silver nanowire networks Nano Lett. 12313844 